\title{
THERMAL INSTABILITY OF INCOMPRESSIBLE NON - NEWTONIAN VISCOELASTIC FLUID WITH THE EFFECT OF ROTATION AND MAGNETIC FIELD
}

\author{
R. Vasantha kumari ${ }^{1}$, R.Sekar $^{2}$, K. Thirumurugan ${ }^{3}$ \\ ${ }^{1}$ Principal, Kasthurba College for Women, Villianur, Puducherry, India, ${ }^{2}$ Professor, Department Of Mathematics, \\ Pondicherry Engineering College, India, ${ }^{3}$ Research - Scholar, Department of Mathematics, Bharathiar University, \\ Coimbatore, India, thirumurugan.kirithish@gmail.com
}

\begin{abstract}
The thermal convection of incompressible Walters' $B$ ' Rotating viscoelastic fluid is considered in the presence of uniform vertical magnetic field. By applying normal mode analysis method, the dispersion relation has been derived and solved analytically. For stationary convection, Walters ' $B$ ' viscoelastic fluid behaves like an ordinary (Newtonian) fluid. It is studied that rotation has a stabilizing effect, whereas the magnetic field has both stabilizing and destabilizing effects. The rotation and magnetic field are found to introduce oscillatory mode in the system which were non - existent in their absence. The results are presented through graphs.
\end{abstract}

Key words: Walters'B' Fluid, thermal convection, viscoelasticity, Rotation, Magnetic field.

\section{INTRODUCTION}

A detailed account of the theoretical and experimental study of thermal instability in Newtonian fluids under varying assumptions of hydrodynamics and hydro magnetics has been given by Chandrasekhar [3]. The thermal instability of a fluid layer with maintained adverse temperature gradient by heating the underside plays an important role in geophysics, interior of the Earth, Oceanography and the atmospheric physics have investigated by Rayleigh [9] and Jeffreys [4]. Bhatia and Steiner [2] have studied the problem of thermal instability of a maxwellian viscoelastic fluid in the presence of the rotation and have found that a rotation has a destabilizing effect, in contrast to the stabilizing effect on Newtonian viscous fluid. Sharma [11] has studied the thermal instability of a layer of oldroydian viscoelastic fluid acted on by a uniform rotation and found that rotation has destabilizing as well as stabilizing effect under certain conditions in contrast to that of a maxwell fluid where it has a destabilizing effect. In another study, Sharma [10] has studied thermal instability in a viscoelastic fluid in the hydromagnetics. Oldroyd [7] investigated non - Newtonian effects in study motion of some idealized elastic - viscous liquids.

Sharma [12] investigated the thermal instability of compressible fluids in the presence of rotation and magnetic field. Kumar et al. [5] have studied the thermal convection in a Walters'(model B') elastico - viscous dusty fluid in hydromagnetics with the effects of compressibility and rotation. In a recent study, Rana and Kango[8] have considered the thermal instability of compressible walters'(model B') rotating fluid in the presence of suspended particles and the porous medium. Kumar et al. [6] have investigated the thermal instability of walters' $\mathrm{B}^{\prime}$ visoelastic fluid permeated with suspended particles in hydromagnetics in porous medium. Walters [14] has studied non - Newtonian effects in some elastico - viscous liquids whose behavior at small rates of shear is characterized by a general linear equation of a state. Sharma et al. [13] has analyzed the thermal instability of walters'(model B') elastico - viscous fluid in the presence of variable gravity field and rotation in porous medium. Recently, Aggarwal and Anushri verma [1] have considered the effect of rotation and the magnetic field on thermal instability of viscoelastic fluid permeated with suspended particles.

There is growing importance of non - Newtonian fluids, convection in fluid layer heated from below, magnetic field, and rotation, the present paper attempts to study the effect of uniform vertical magnetic field on Non - Newtonian viscoelastic fluid heated from below in the presence of a uniform rotation.

\section{MATHEMATICAL MODEL AND PERTURBATION EQUATIONS}

Consider an infinite, horizontal, incompressible electrically conducting Walters'B' viscoelastic fluid layer of thickness d, heated from below so that the temperatures and densities at the bottom surface $\mathrm{z}=0$ are $\mathrm{T} 0$ and $\rho 0$ and at the upper surface $\mathrm{z}$ $=\mathrm{d}$ are $\mathrm{Td}$ and $\rho \mathrm{d}$, respectively, and that a uniform temperature gradient $\beta(=|\mathrm{dT} / \mathrm{dz}|)$ is maintained. The gravity 
field $\mathrm{g}(0,0,-\mathrm{g})$, a uniform vertical magnetic field $\mathrm{H}(0,0, \mathrm{H})$, and a uniform vertical rotation $\Omega(0,0, \Omega)$ act on the system.

Let $q(u, v, w), p, \rho, T, v$, and $v^{\prime}$ denote the velocity, pressure, density, temperature, kinematic viscosity, and kinematic viscoelasticity, respectively, and $\mathrm{r}(\mathrm{x}, \mathrm{y}, \mathrm{z})$.

The equations of motion, continuity, heat conduction, and Maxwell's equations governing the flow of Walters' $\mathrm{B}^{\prime}$ viscoelastic fluid in the presence of magnetic field and rotation are

$\frac{\partial \mathrm{q}}{\partial \mathrm{t}}+(\mathrm{q} . \nabla) \mathrm{q}=-\nabla\left(\frac{\mathrm{p}}{\rho_{0}}-\frac{1}{2}|\Omega \times \mathrm{r}|^{2}\right)+\mathrm{g}\left(1+\frac{\delta \rho}{\rho_{0}}\right)+$

$\left(\mathrm{v}-\mathrm{v}^{\prime} \frac{\partial}{\partial \mathrm{t}}\right) \nabla^{2} \mathrm{q}+\frac{\mu_{\mathrm{e}}}{4 \pi \rho_{0}}(\nabla \times \mathrm{H}) \times \mathrm{H}+2(\mathrm{q} \times \Omega)$,

$\nabla \cdot q=0$,

$\frac{\partial \mathrm{T}}{\partial \mathrm{t}}+(\mathrm{q} \times \nabla) \mathrm{T}=\chi \nabla^{2} \mathrm{~T}$,

$\nabla . \mathrm{H}=0$,

$\frac{\partial \mathrm{H}}{\partial \mathrm{t}}=(\mathrm{H} . \nabla) \mathrm{q}+\eta \nabla^{2} \mathrm{H}$

The equation of the fluid state is

$\rho=\rho 0[1-\alpha(\mathrm{T}-\mathrm{T} 0)]$,

where $\rho 0$, T0 are, respectively, the density and temperature of the fluid at the reference level $z=0$ and $\alpha$ is the coefficient of thermal expansion. From equation (1), we made use of the Boussinesq approximation, which states that the density variations are ignored in all terms in the equations of motion except the external force term. The magnetic permeability $\mu$, thermal diffusivity $\chi$, and electrical resistivity $\eta$ are all assumed to be constant.

The basic state is one in which the velocity, density, pressure, and temperature at any point in the fluid are, respectively, given by

$q=(0,0,0), \quad \rho=\rho(z), \quad p=p(z), \quad T=T(z)$

The change in density $\delta \rho$, caused by the perturbation $\theta$ in temperature, is given by

$$
\delta \rho=-\alpha \rho 0 \theta
$$

Then the linearized perturbation equations are

$$
\begin{gathered}
\frac{\partial \mathrm{q}}{\partial \mathrm{t}}=\frac{-1}{\rho_{0}}(\nabla \delta \mathrm{p})-\mathrm{ga} \Theta+\left(\mathrm{v}-\mathrm{v}^{\prime} \frac{\partial}{\partial \mathrm{t}}\right) \nabla^{2} \mathrm{q}+\frac{\mu_{\mathrm{e}}}{4 \pi \rho_{0}}(\nabla \times \mathrm{h}) \times \mathrm{H} \\
+2(\mathrm{q} \times \Omega)
\end{gathered}
$$

$\frac{\partial \theta}{\partial t}=\beta w+\chi \nabla^{2} \theta$

$\nabla \cdot h=0$,

$\frac{\partial \mathrm{h}}{\partial \mathrm{t}}=(\mathrm{H} . \nabla) \mathrm{q}+\eta \nabla^{2} \mathrm{~h}$

Where $\mathrm{q}(\mathrm{u}, \mathrm{v}, \mathrm{w}), \quad \delta \mathrm{p}, \quad \delta \rho, \quad \theta$, and $\mathrm{h}(\mathrm{hx}, \mathrm{hy}, \mathrm{hz})$ denote, respectively, the perturbations in velocity q (initially zero), pressure $p$, density $\rho$, temperature $T$, and the magnetic field $\mathrm{H}(0,0, \mathrm{H})$. Within the framework of Boussinesq approximation,(9) Obtains

$$
\begin{aligned}
& \frac{\partial}{\partial \mathrm{t}} \nabla^{2}=\left(\mathrm{v}-\mathrm{v}^{\prime} \frac{\partial}{\partial \mathrm{t}}\right) \nabla^{4} \mathrm{w}+\frac{\mu_{\mathrm{e}}}{4 \pi \rho_{0}} \nabla^{2}\left(\frac{\partial \mathrm{h}_{\mathrm{z}}}{\partial \mathrm{z}}\right)+\mathrm{g} \alpha\left(\frac{\partial^{2} \theta}{\partial \mathrm{x}^{2}}+\frac{\partial^{2} \theta}{\partial \mathrm{y}^{2}}\right)- \\
& 2 \Omega \frac{\partial \zeta}{\partial \mathrm{z}^{\prime}} \\
& \frac{\partial \zeta}{\partial \mathrm{z}}=\left(\mathrm{v}-\mathrm{v}^{\prime} \frac{\partial}{\partial \mathrm{t}}\right) \nabla^{2} \zeta+2 \Omega \frac{\partial \mathrm{w}}{\partial \mathrm{z}}-\frac{\mu_{\mathrm{e}} \mathrm{H}}{4 \pi \rho_{0}} \frac{\partial \xi}{\partial \mathrm{z}} \\
& \left(\frac{\partial}{\partial \mathrm{t}}-\chi \nabla^{2}\right) \theta=\beta \mathrm{w} \\
& \quad\left(\frac{\partial}{\partial \mathrm{t}}-\eta \nabla^{2}\right) \mathrm{h}_{\mathrm{z}}=\mathrm{H} \frac{\partial \mathrm{w}}{\partial \mathrm{z}} \\
& {\left[\frac{\partial}{\partial \mathrm{t}}-\eta \nabla^{2}\right] \xi=\mathrm{H} \frac{\delta \zeta}{\delta \mathrm{x}}}
\end{aligned}
$$

where $\nabla 2=\partial 2 / \partial \mathrm{x} 2+\partial 2 / \partial \mathrm{y} 2+\partial 2 / \partial \mathrm{z} 2$ and $\zeta=\partial \mathrm{v} / \partial \mathrm{x}-\partial \mathrm{u} / \partial \mathrm{y} ; \xi$ $=\partial \mathrm{hy} / \partial \mathrm{x}-\partial \mathrm{hx} / \partial \mathrm{y}$ stand for the z-components of vorticity and current density, respectively.

\section{THE DISPERSION RELATION}

We now analyze the disturbances into normal modes, assuming that the perturbation quantities are of the form

$[\mathrm{w}, \theta, \mathrm{hz}, \zeta, \xi]=[\mathrm{w}(\mathrm{z}), \Theta(\mathrm{z}), \mathrm{K}(\mathrm{z}), \mathrm{Z}(\mathrm{z}), \mathrm{X}(\mathrm{z})] \exp (\mathrm{ikx} \mathrm{x}+$ ikyy+nt),

where $\mathrm{kx}, \mathrm{ky}$ are the wave numbers along $\mathrm{x}$ and $\mathrm{y}$ directions, respectively, $\mathrm{k}=(\mathrm{kx} 2+\mathrm{ky} 2) 1 / 2$ is the resultant wave number, and $\mathrm{n}$ is the growth rate which is, in general, a complex constant.

Using equation (11), (12) in non-dimensional form transform to

$$
\begin{aligned}
& {\left[\sigma\left(D^{2}-\mathrm{a}^{2}\right)^{2} \mathrm{~W}+\left(\frac{\mathrm{gad}^{2}}{\mathrm{v}}\right) \mathrm{a}^{2} \Theta+\frac{2 \Omega \mathrm{d}^{3}}{\mathrm{v}} \mathrm{DZ}-\frac{\mu_{\mathrm{e}} \mathrm{Hd}}{4 \pi \rho_{0} \mathrm{v}}\left(\mathrm{D}^{2}-\right.\right.} \\
& \mathrm{a} 2 \mathrm{DK}=1-\mathrm{F} \sigma(\mathrm{D} 2-\mathrm{a} 2) 2 \mathrm{~W}, \\
& \left.\left[(1-\mathrm{F} \sigma)\left(\mathrm{D}^{2}-\mathrm{a}^{2}\right)-\sigma\right] \mathrm{Z}=-\left(\frac{2 \Omega \mathrm{d}}{\mathrm{v}}\right) \mathrm{DW}-\frac{\mu_{\mathrm{e}} \mathrm{Hd}}{4 \pi \rho_{0}} \mathrm{DX}\right],(13) \\
& {\left[\mathrm{D}^{2}-\mathrm{a}^{2}-\mathrm{p}_{1} \sigma\right] \Theta=-\left(\frac{\beta \mathrm{d}^{2}}{\chi}\right) \mathrm{W}}
\end{aligned}
$$

$\nabla \cdot q=0$ 
$\left[D^{2}-\mathrm{a}^{2}-\mathrm{p}_{2} \sigma\right] \mathrm{k}=-\left(\frac{\mathrm{Hd}}{\eta}\right) \mathrm{DW}$

$\left[D^{2}-a^{2}-p_{2} \sigma\right] X=-\left(\frac{H d}{\eta}\right) D Z$

Where we have we have put $\mathrm{a}=\mathrm{kd}, \sigma=\mathrm{nd} 2 / \mathrm{v}, \mathrm{F}=\mathrm{v}^{\prime} / \mathrm{d} 2 ; \mathrm{p} 1=$ $v / \chi$ is the Prandtl number and $p 2=v / \eta$ is the magnetic Prandtl number and $\left(\mathrm{x}^{\prime}, \mathrm{y}^{\prime}, \mathrm{z}^{\prime}\right)=(\mathrm{x} / \mathrm{d}, \mathrm{y} / \mathrm{d}, \mathrm{z} / \mathrm{d}), \mathrm{D}=\mathrm{d} / \mathrm{dz}$. For convenience, the dashes are dropped

Consider the case where both the boundaries are free as well as perfect conductors of heat, while the adjoining medium is also perfectly conducting. The case of two free boundaries is slightly artificial, except in stellar atmospheres and in certain geophysical situations where it is most appropriate. However, the case of two free boundaries allows us to obtain analytical solution without affecting the essential features of the problem. The appropriate boundary conditions, with respect to which (12) - (16) must be solved, are

$\mathrm{W}=\mathrm{D} 2 \mathrm{~W}=0, \quad \mathrm{DZ}=0, \quad \Theta=0 \quad$ at $\mathrm{z}=0, \mathrm{z}=1$,

$\mathrm{DX}=0, \mathrm{~K}=0$, on a perfectly conducting boundary.

Using the above boundary conditions, it can be shown that all the even-order derivatives of $\mathrm{W}$ must vanish for $\mathrm{z}=0$ and $\mathrm{z}=$ 1 , and hence the proper solution of $\mathrm{W}$ characterizing the lowest mode is

$\mathrm{W}=\mathrm{W} 0 \sin \pi \mathrm{z}, \quad$ where $\mathrm{W} 0$ is a constant.

Eliminating $\Theta, \quad K, \quad Z$, and $X$ between(12)-(16) and substituting(18) in the resultant equation, we obtain the dispersion relation

$$
\begin{aligned}
\mathrm{R}_{1}=\left(\frac{1+\mathrm{x}}{\mathrm{x}}\right) \frac{\left[\left\{\left(1-\mathrm{i} \mathrm{F}_{1} \sigma_{1} \pi^{2}\right)(1+\mathrm{x})+\mathrm{i} \sigma_{1}\right\}\left\{1+\mathrm{x}+\mathrm{i} \sigma_{1} \mathrm{p}_{2}\right\}+\mathrm{Q}_{1}\right]\left[1+\mathrm{x}+\mathrm{i} \sigma_{1} \mathrm{p}_{1}\right]}{\left(1+\mathrm{x}+\mathrm{i} \sigma_{1} \mathrm{p}_{2}\right)} \\
+\frac{\mathrm{T}_{1}\left(1+\mathrm{x}+\mathrm{i} \sigma_{1} \mathrm{p}_{2}\right)\left(1+\mathrm{x}+\mathrm{i} \sigma_{1} \mathrm{p}_{1}\right)}{\left\{\mathrm{x}\left[\left\{\left(1-\mathrm{i} \mathrm{F}_{1} \sigma_{1} \pi^{2}\right)(1+\mathrm{x})+\mathrm{i} \sigma_{1}\right\}\left\{1+\mathrm{x}+\mathrm{i} \sigma_{1} \mathrm{p}_{2}\right\}+\mathrm{Q}_{1}\right]\right\}}
\end{aligned}
$$

Where $\mathrm{R}=\operatorname{g\alpha } \beta \mathrm{d} 4 / v \chi, \mathrm{Q}=\mu \mathrm{eH} 2 \mathrm{~d} 2 / 4 \pi \rho 0 v \eta, \mathrm{Ta}=4 \Omega 2 \mathrm{~d} 4 / \mathrm{v} 2$ stand for the Rayleigh-number, the Chandrasekhar number, the Taylor number, respectively. Putting

$x=\frac{a^{2}}{\pi^{2}}, \quad R_{1}=\frac{R}{\pi^{4}}, \quad i \sigma_{1}=\frac{\sigma}{\pi^{2}}, \quad F_{1}=\pi^{2} F$

$\mathrm{T}_{1}=\frac{\mathrm{T}_{\mathrm{A}}}{\pi^{4}}, \quad \mathrm{Q}_{1}=\frac{\mathrm{Q}}{\pi^{2}}, \quad \mathrm{i}=\sqrt{-1}$,

\section{THE STATIONARY CONVECTION}

When the instability sets in as stationary convection, the marginal state will be characterized by $\sigma=0$. Putting $\sigma=0$, the dispersion relation (19) reduces to

$$
\mathrm{R}_{1}=\left(\frac{1+\mathrm{x}}{\mathrm{x}}\right)\left[(1+\mathrm{x})^{2}+\mathrm{Q}_{1}\right]+\frac{\mathrm{T}_{1}(1+\mathrm{x})^{2}}{\mathrm{x}\left[(1+\mathrm{x})^{2}+\mathrm{Q}_{1}\right]}
$$

We thus find that for the stationary convection, the viscoelasticity parameter $F$ vanishes with $\sigma$ and Walters' $B$ ' viscoelastic fluid behaves like an ordinary Newtonian fluid. To study the effects of rotation and magnetic field, we examine the natures of and $\frac{\mathrm{dR}_{1}}{\mathrm{dQ}_{1}}$ analytically.

Equation (21) yields

$\frac{\mathrm{dR}_{1}}{\mathrm{dT}_{1}}=\frac{(1+\mathrm{x})^{2}}{\mathrm{x}\left[(1+\mathrm{x})^{2}+\mathrm{Q}_{1}\right]}$

$\frac{\mathrm{dR}_{1}}{\mathrm{~d} \mathrm{Q}_{1}}=\frac{(1+\mathrm{x})}{\mathrm{x}}-\frac{\mathrm{T}_{1}(1+\mathrm{x})^{2}}{\mathrm{x}\left[(1+\mathrm{x})^{2}+\mathrm{Q}_{1}\right]^{2}}$

It is evident from (22) that for a stationary convection, $\frac{\mathrm{dR}_{1}}{\mathrm{dT}_{1}}$ is always positive, thus, the rotation has a stabilizing effect on the system.

\section{Magnetic field vs. Rayleigh Number}

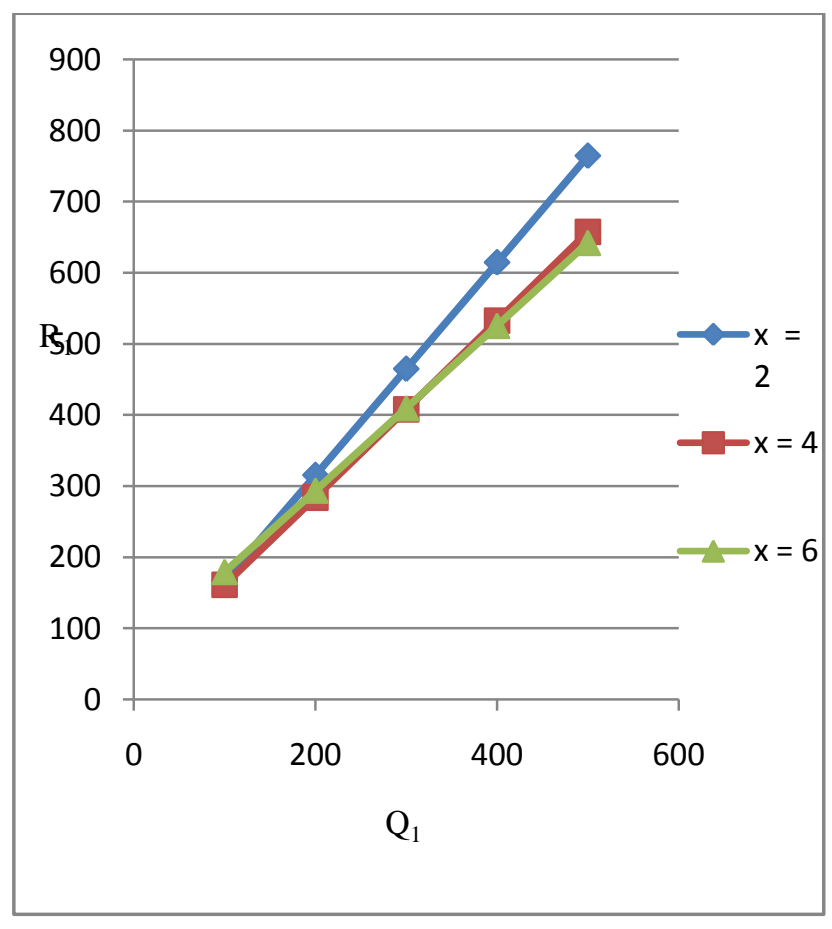

Figure 1 The Variation of R1 With Q1 for fixed values of T1 $=100$ and $\mathrm{x}=2,4,6$ 


\section{Rotation vs. Rayleigh Number}

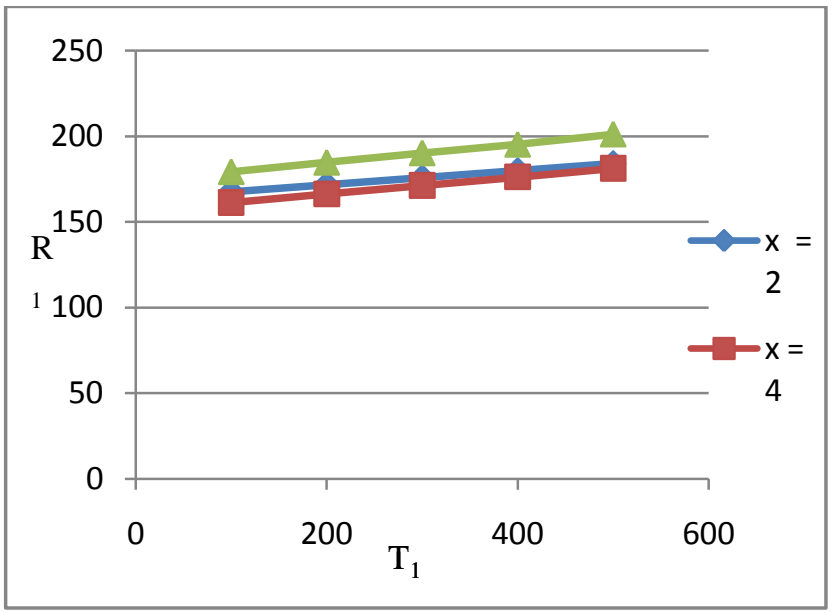

Figure 2 The Variation of R1 With T1 for fixed values of Q1 $=100$ and $\mathrm{x}=2,4,6$

It is clear from (23) for a stationary convection, dR1/dQ1 may be positive as well as negative, thus, the magnetic field has both stabilizing and destabilizing effects on the system.

The dispersion relation (21) is also analyzed numerically. Figure (1) shows the variation of R1 with respect to Q1, for fixed value of $\mathrm{T} 1=100$ and wave numbers $\mathrm{x}=2,4,6$. In Figure (2), R1 is plotted against $\mathrm{T} 1$, for fixed value of $\mathrm{Q} 1=$ 100 and wave numbers $x=2,4,6$. The Rayleigh number R1 increases with increase in rotation parameter $\mathrm{T} 1$ showing its stabilizing effect on the system. . It clearly depicts both the stabilizing and destabilizing effects of the magnetic field on the system.

\section{STABILITY OF THE SYSTEM AND OSCILLATORY MODES}

Multiplying (12) by $\mathrm{W} *$, the complex conjugate of $\mathrm{W}$, integrating over the range of $\mathrm{z}$ and using (13)-(16), together with the boundary conditions (17), We obtain

$$
\begin{array}{r}
-\sigma I_{1}+\frac{\text { gaxa }^{2}}{v \beta}\left(I_{2}+p_{1} \sigma^{*} I_{3}\right)-d^{2}\left(1-F \sigma^{*}\right) I_{4}-d^{2} \sigma^{*} I_{5} \\
-\frac{\mu_{\mathrm{e}}{ }^{2} \eta}{4 \pi \rho_{0} v}\left(I_{6}+p_{2} \sigma I_{7}\right)-\frac{\mu_{e} \eta}{4 \pi \rho_{0} v}\left(I_{8}+p_{2} \sigma^{*} I_{9}\right)(I-F \sigma) I_{10},(24)
\end{array}
$$

Where,

$$
\begin{aligned}
& I_{1}=\int_{0}^{1}\left(|D W|^{2}+a^{2}|W|^{2}\right) d z, \quad I_{2}=\int_{0}^{1}\left(|D \Theta|^{2}+a^{2}|\Theta|^{2}\right) d z, \\
& I_{3}=\int_{0}^{1}\left(|\Theta|^{2}\right) d z, \quad I_{4}=\int_{0}^{1}\left(|D Z|^{2}+a^{2}|Z|^{2}\right) d z, \\
& I_{5}=\int_{0}^{1}\left(|Z|^{2}\right) d z, \quad I_{6}=\int_{0}^{1}\left(|D X|^{2}+a^{2}|X|^{2}\right) d z,
\end{aligned}
$$

$$
\begin{aligned}
& \mathrm{I}_{7}=\int_{0}^{1}\left(|\mathrm{X}|^{2}\right) \mathrm{dz}, \quad \mathrm{I}_{8}=\int_{0}^{1}\left(\left|\mathrm{D}^{2} \mathrm{~K}\right|^{2}+2 \mathrm{a}^{2}|\mathrm{DK}|^{2}+\right. \\
& \left.\mathrm{a}^{4}|\mathrm{~K}|^{2}\right) \mathrm{dz}, \\
& \mathrm{I}_{9}=\int_{0}^{1}\left(|\mathrm{DK}|^{2}+\mathrm{a}^{2}|\mathrm{~K}|^{2}\right) \mathrm{dz}, \\
& \mathrm{I}_{10}=\int_{0}^{1}\left(\left|\mathrm{D}^{2} \mathrm{~W}\right|^{2}+2 \mathrm{a}^{2}|\mathrm{DW}|^{2}+\mathrm{a}^{4}|\mathrm{~W}|^{2}\right) \mathrm{dz},
\end{aligned}
$$

and $\sigma *$ is the complex conjugate of $\sigma$. The integrals I1......I10 are all positive definite. Putting

$\sigma=\sigma r+i \sigma i$, where $\sigma r$, $\sigma i$ are real. Separating the real and imaginary parts of (24), we obtain

$$
\begin{aligned}
& \sigma_{\mathrm{r}}\left[-\mathrm{I}_{1}+\frac{\mathrm{ga \chi \textrm {a } ^ { 2 }}}{\mathrm{v} \beta} \mathrm{p}_{1} \mathrm{I}_{3}-\mathrm{d}^{2} \mathrm{FI}_{4}-\mathrm{d}^{2} \mathrm{I}_{5}-\frac{\mu_{\mathrm{e}} \mathrm{d}^{2} \eta}{4 \pi \rho_{0} \mathrm{v}} \mathrm{p}_{2}-\right. \\
& \left.\frac{\mu_{\mathrm{e}} \eta}{4 \pi \rho_{0} \mathrm{v}} \mathrm{p}_{2} \mathrm{I}_{9}-\mathrm{FI}_{10}\right] \\
& =-\frac{g a a^{2}}{v \beta} I_{2}+d^{2} I_{4}+\frac{\mu_{e} d^{2} \eta}{4 \pi \rho_{0} v} I_{6}+\frac{\mu_{e} \eta}{4 \pi \rho_{0} v} I_{8}+I_{10}, \\
& \sigma_{i}\left[I_{1}+\frac{\text { gaxa }}{v \beta} p_{1} I_{3}-d^{2} F_{4}-d^{2} I_{5}-\frac{\mu_{e} d^{2} \eta}{4 \pi \rho_{0} v} p_{2} I_{7}-\right. \\
& \mu \mathrm{e \eta} 4 \pi \rho 0 \mathrm{vp} 2 \mathrm{I} 9+\mathrm{FI} 10=0 \text {, }
\end{aligned}
$$

From (27), it is clear that $\sigma \mathrm{i}$ is zero when the quantity multiplying it is not zero and arbitrary when this quantity is zero. If $\sigma i \neq 0$, then $(27)$ gives

$\frac{\text { ga } \mathrm{a}^{2}}{\mathrm{v} \beta} \mathrm{p}_{1} \mathrm{I}_{3}-\mathrm{d}^{2} \mathrm{FI}_{4}-\mathrm{d}^{2} \mathrm{I}_{5}-\frac{\mu_{\mathrm{e}} \eta}{4 \pi \rho_{0} \mathrm{v}} \mathrm{p}_{2} \mathrm{I}_{9}=-\mathrm{I}_{1}-\frac{\mu_{\mathrm{e}} \mathrm{d}^{2} \eta}{4 \pi \rho_{0} \mathrm{v}} \mathrm{p}_{2} \mathrm{I}_{7}-$ $\mathrm{FI}_{10}$,

Substituting in (26), we have

$$
\begin{aligned}
& \mathrm{I}_{10}+\frac{\mu_{\mathrm{e}} \eta}{4 \pi \rho_{0} \mathrm{v}} \mathrm{I}_{8}+\frac{\mu_{\mathrm{e}} \eta \mathrm{d}^{2}}{4 \pi \rho_{0} \mathrm{v}} \mathrm{I}_{6}+\mathrm{d}^{2} \mathrm{I}_{4}+2 \sigma_{\mathrm{r}}\left[\mathrm{I}_{1}+\frac{\mu_{\mathrm{e}} \mathrm{d}^{2} \eta}{4 \pi \rho_{0} \mathrm{v}} \mathrm{p}_{2} \mathrm{I}_{7}+\right. \\
& \text { FI10 }=\text { ga } 2 \mathrm{a} 2 \mathrm{v} \beta \mathrm{I} 2
\end{aligned}
$$

On applying Rayleigh-Ritz inequality, Equation (29) gives

$\frac{\left(\pi^{2}+a^{2}\right)^{3}}{a^{2}} \int_{0}^{1}|w|^{2} d z+\frac{\left(\pi^{2}+a^{2}\right)}{a^{2}} \times\left\{\frac{\mu_{e} \eta}{4 \pi \rho_{0} v} I_{8}+\frac{\mu_{e} d^{2} \eta}{4 \pi \rho_{0} v} I_{6}+\right.$ $\mathrm{d} 2 \mathrm{I} 4+2 \sigma r \mathrm{I} 1+\mu \mathrm{ed} 2 \eta 4 \pi \rho 0 \mathrm{vp} 2 \mathrm{I} 7+\mathrm{FI} 10 \leq \operatorname{ga\chi v} \beta 01 \mathrm{w} 2 \mathrm{dz}$, (30)

Hence equation (30) yields

$$
\begin{aligned}
& {\left[\frac{27 \pi^{4}}{4}-\frac{\text { gax }}{v \beta}\right] \int_{0}^{1}|w|^{2} d z+\frac{\left(\pi^{2}+a^{2}\right)}{a^{2}} \times\left\{\frac{\mu_{e} \eta}{4 \pi \rho_{0} v} I_{8}+\frac{\mu_{e} d^{2} \eta}{4 \pi \rho_{0} v} I_{6}+\right.} \\
& d 2 I 4+2 \sigma r I 1+\mu e d 2 \eta 4 \pi \rho 0 v p 2 I 7+F I 10 \leq 0
\end{aligned}
$$

since minimum value of $(\pi 2+\mathrm{a} 2) 3 / \mathrm{a} 2$ with respect to a2 is $27 \pi 4 / 4$. Now, let $\sigma r \geq 0$, we necessarily have from (31) that 
$\frac{27 \pi^{4}}{4}<\frac{\text { ga } \chi}{v \beta}$

Hence if

$$
\frac{27 \pi^{4}}{4} \geq \frac{\operatorname{gax}}{\mathrm{v} \beta}
$$

then $\sigma r<0$. Therefore, the system is stable.

The system is stable under condition (33) and also the system gets unstable under condition (32). In the absence of rotation and magnetic field, (27) reduces to

$\sigma_{\mathrm{i}}\left[\mathrm{I}_{1}+\frac{\mathrm{gax \textrm {a } ^ { 2 }}}{\mathrm{v} \beta} \mathrm{p}_{1} \mathrm{I}_{3}+\mathrm{FI}_{10}\right]=0$

and the terms in brackets are positive definite. Thus, $\sigma \mathrm{i}=0$, which means that oscillatory modes are not allowed and the principle of exchange of stabilities is valid. The viscoelasticity, magnetic field and rotation introduce oscillatory modes (as $\sigma \mathrm{i}$ may not be zero) in the system which were non-existent in their absence.

\section{CONCLUSIONS}

Combined effect of various parameters that is magnetic field, rotation and incompressibility has been investigated on thermal instability of a walters'B' fluid. The main results from the analysis of the paper are:

i. Stationary conversion, walters'B' fluid behaves like an ordinary Newtonian fluid due to the vanishing of the viscoelastic parameter.

ii. To study the effects of rotation and magnetic field, we examine the nature of $\frac{d R_{1}}{d Q_{1}}$ analytically. It has been found the rotation is stabilizing effect of the system, whereas the magnetic field has both stabilizing and destabilizing effects.

iii. The magnetic field, rotation and viscoelasticity introduce oscillatory modes in the system which were non-existent in their absence.

\section{REFERENCES}

[1] A. K. Aggarwal and Anushri verma, wseas transactions on mathematics, 2010,8(9), 593-602.

[2] P.K Bhatia and J.M. Steiner, Z. Angew, math.mech(1972), $52,321-327$.

[3] S.Chandrasekhar, Hydrodynamic and hydromagnetic stability, dover publication, New York, 1981.

[4] H.Jeffreys, Philosophical magazine 1926, 2, 833 - 844.

[5]. V. Kumar, A.K. Aggarwal and S.Kumar, Int. J. Appl Mech. Eng, 2010, 15, 1, 51- 62.

[6] P. Kumar , G.J. Singh and R.Lal, Thermal Science, 2004, $18,1,51-61$

[7] J.G. Oldroyd, Proc. Roy. Soc. London, 1958, A245, 278 297.
[8] G.C. Rana and S.K Kango, Adv.Appl.Sci.Res 2011, 2, 3, $586-597$.

[9] L.Rayleigh, philosophical magazine, 1916, 32, 529-546.

[10] R.C. Sharma, Acta Physics Hungeria, 1975 , 38, 293 298.

[11] R.C. Sharma, Acta Physics Hungeria, 1976 , 40, 11 - 17.

[12] R.C. Sharma, J.math.anal.Appl.,, 1977 , 38, 293 - 298.

[13]. V. Sharma and G.C. Rana, J. Non-Equilib thermodyn. 2001, 26, 31 .

[14] K. Walters, Quart. J.Mech. Appl.Math. 1962,15,76 - 96. 